\title{
QTL mapping for starch paste viscosity of rice (Oryza sativa L.) using chromosome segment substitution lines derived from two sequenced cultivars with the same $W x$ allele
}

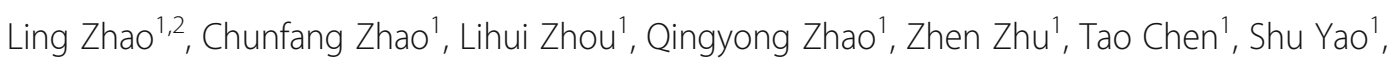
Yadong Zhang ${ }^{1 *}$ and Cailin Wang ${ }^{1,2^{*}}$ (D)

\begin{abstract}
Background: The eating and cooking qualities (ECQs) of rice (Oryza sativa L.) are key characteristics affecting variety adoption and market value. Starch viscosity profiles tested by a rapid visco analyzer (RVA) offer a direct measure of ECQs and represent the changes in viscosity associated with starch gelatinization. RVA profiles of rice are controlled by a complex genetic system and are also affected by the environment. Although Waxy $(W x)$ is the major gene controlling amylose content (AC) and ECQs, there are still other unknown genetic factors that affect ECQs.

Results: Quantitative trait loci (QTLs) for starch paste viscosity in rice were analyzed using chromosome segment substitution lines (CSSLS) developed from the two cultivars 9311 and Nipponbare, which have same Wx-b allele. Thus, the effect of the major locus Wx was eliminated and the other locus associated with the RVA profile could be identified. QTLs for seven parameters of the starch RVA profile were tested over four years in Nanjing, China. A total of 310 QTLs were identified (from 1 to 55 QTLs per trait) and 136 QTLs were identified in more than one year. Among them, 6 QTLs were stalely detected in four years and 26 QTLs were detected in at least three years including 13 pleiotropic loci, controlling 2 to 6 RVA properties simultaneously. These stable QTL hotspots were colocated with several known starch synthesis-related genes (SSRGs). Sequence alignments showed that nucleotide and amino acid sequences of most SSRGs were different between the two parents. Finally, we detected stable QTLs associated with multiple starch viscosity traits near $W x$ itself, supporting the notion that additional QTLs near Wx control multiple characteristic values of starch viscosity.
\end{abstract}

\footnotetext{
*Correspondence: zhangyd@jaas.ac.cn; cailin_wang@163.com

Institute of Food Crops, Jiangsu Academy of Agricultural Sciences, Jiangsu High Quality Rice R\&D Center, Nanjing Branch of China National Center for Rice Improvement, Nanjing 210014, China

Full list of author information is available at the end of the article
}

(c) The Author(s). 2021 Open Access This article is licensed under a Creative Commons Attribution 4.0 International License, which permits use, sharing, adaptation, distribution and reproduction in any medium or format, as long as you give appropriate credit to the original author(s) and the source, provide a link to the Creative Commons licence, and indicate if changes were made. The images or other third party material in this article are included in the article's Creative Commons licence, unless indicated otherwise in a credit line to the material. If material is not included in the article's Creative Commons licence and your intended use is not permitted by statutory regulation or exceeds the permitted use, you will need to obtain permission directly from the copyright holder. To view a copy of this licence, visit http://creativecommons.org/licenses/by/4.0/ The Creative Commons Public Domain Dedication waiver (http://creativecommons.org/publicdomain/zero/1.0/) applies to the data made available in this article, unless otherwise stated in a credit line to the data. 
Conclusions: By eliminating the contribution from the major locus WX, multiple QTLs associated with the RVA profile of rice were identified, several of which were stably detected over four years. The complexity of the genetic basis of rice starch viscosity traits might be due to their pleiotropic effects and the multiple QTL hot spots. Minor QTLs controlling starch viscosity traits were identified by using the chromosome segment substitution strategy. Allele polymorphism might be the reason that QTLs controlling RVA profile characteristics were detected in some known SSRG regions.

Keywords: Rice (Oryza sativa L.), Starch paste viscosity, Quantitative trait locus, Chromosome segment substitution lines

\section{Background}

Rice (Oryza sativa L.) is one of the most important cereal crops worldwide, with about half of the world's population consuming it as a main source of dietary calories [1]. Grain quality is a key factor affecting rice production and market value. Current breeding programs aim to improve rice quality particularly with respect to eating and cooking qualities (ECQs).

As the main chemical component of grains, starch and its fine structure determine rice ECQs [2]. The starch viscosity profile is typically tested with a rapid visco analyzer (RVA) and offers a direct measure of ECQs by characterizing the pasting behavior and measuring the changes in viscosity associated with starch gelatinization during cooking. In recent years, starch RVA profiles have become an increasingly popular measure of rice quality, as they are easy to perform and only small samples are required [3]. Indeed, starch RVA profiles have been used as a novel tool to estimate ECQs, and some models were developed successfully in breeding programs $[2,4,5]$.

Starch RVA profiles of rice grains are controlled by a complex genetic system that involves multiple quantitative trait loci (QTLs), several with major effects on the trait, and many starch synthesis-related genes (SSRGs) [6]. Linkage mapping using different populations identified a major QTL in the genomic region that contains the gene $\operatorname{Waxy}(W x)$ on chromosome 6 . Wx encodes the granule-bound starch synthase (GBSS) that is mainly responsible for longer amylose chains [7-9]. Many additional loci associated with starch RVA profiles were also shown to affect the ECQs of rice [10]. The alkali degeneration gene locus $(A L K)$ on chromosome 6 encoding starch synthase II a (SSII-3), is the major factor responsible for gelatinization temperature (GT) and the distribution of amylopectin chain length [11-13]. Other SSRGs, such as genes encoding debranching enzyme (DBE), isoamylases (ISA), starch branching enzyme (SBE), soluble starch synthase (SSS), and pullulanase (PUL), play minor effects on starch RVA profiles [14]. These SSRGs have widely been proposed to control amylose content $(\mathrm{AC}), \mathrm{GT}$, or certain other rice quality traits [15-17].
The heredity of starch RVA profile characters is complex and could easily be effected by environmental factors, especially the temperature during the grain filling stage $[18,19]$. This brings more difficulty in mapping QTLs of consistent RVA traits. Over 200 starch RVA traits-related QTLs have been identified in various mapping populations [3, 7, 18-25]. A few QTLs have been cloned or finely mapped (http://www.gramene.org/, http://www.ricedata.cn/). Certain varieties carrying the same $W x$ allele exhibit different ECQs, indicating that other minor QTLs affect ECQs. Identifying these minor QTLs will be critical to precisely modulate rice grain quality in the future. However, because the effects of minor QTLs might be covered by major QTLs such as $W x$, fine mapping and the cloning of the underlying locus can be challenging. Populations derived from two parents with similar AC or with the same $W x$ allele have been developed to eliminate the effects of $W x$ and detect minor QTLs for starch RVA and ECQs of rice [23, 24, $26,27]$.

The genetic basis of complex traits in rice and the contribution of single-nucleotide polymorphisms have been explored by genome-wide association studies, allowing the generation of testable hypotheses relating allele variation and starch RVA traits [28]. Allelic diversity of SSRGs can explain the genetic basis for the observed phenotypic diversity in starch physicochemical properties across germplasm [14, 17]. However, the distribution of functional alleles is strongly correlated with population structure, which can lead to false results if the population is small [29]. As one of the secondly populations, chromosome segment substitution lines (CSSLs) are very useful for precisely mapping QTLs and dissecting the genetic basis of complex traits [30]. A set of CSSLs had been developed which derived from two sequenced rice cultivars, the indica variety 9311 (recipient) and the japonica variety Nipponbare (donor) [31-33]. Since both cultivars carry the same $W x-b$ allele [22], this CSSLs could be used as an excellent mapping population to detect new QTLs that might be covered by the major $W x$ locus.

In this study, we aimed to detect stable QTLs in the absence of variation at the $W x$ locus for parameters of starch RVA profiles using CSSLs. Our results establish 
the foundation for fine mapping and subsequent cloning of genes responsible for these QTLs, which will help to improve ECQs in rice by molecular breeding.

\section{Results}

Performance of starch RVA profiles in parents and CSSLs in different years

The two parents 9311 and Nipponbare showed similar apparent amylose content (AAC), $15.5 \% \pm 0.2$ and $14.6 \% \pm 0.2 \%$, respectively, in Nanjing from 2017 to 2018. The AAC of CSSLs ranged from 13.1 to $17.2 \%$, with an average AAC of $14.92 \%$ in 2017. The starch RVA profiles of 9311 and Nipponbare differed significantly over four years of the study. Most RVA parameters varied greatly over four years, with the exception of peak time $(\mathrm{PeT})$ and pasting temperature $(\mathrm{PaT})$, which were less affected by environment (Table 1). For indica rice 9311, almost all parameters were much smaller than those in Nipponbare, with the exception of setback viscosity (SB) and PeT.

The starch RVA profiles showed a continuous distribution in the CSSL population over the different years, with the exception of $\mathrm{PaT}$, which exhibited a doublepeak distribution (Fig. 1). The maximum value of peak viscosity (PV) was greater than the other parameters as consequence of greater kurtosis. The starch RVA profiles of CSSLs showed a partial separation for some parameters, such as $\mathrm{PV}$, final viscosity (FV), and $\mathrm{PaT}$, in 2016 and 2017. Among the CSSLs, the phenotypic values of PV, FV, and PaT exhibited a greater range of variation than other traits over four years, whereas breakdown viscosity (BD), SB, and PeT varied little. The mean values for the various RVA parameters of the CSSLs were near the mid-parent value, but some values were not

Table 1 Phenotypic variations of starch RNA characteristics among 9311, Nipponbare, and their CSSL populations

\begin{tabular}{|c|c|c|c|c|c|c|c|}
\hline \multirow[t]{2}{*}{ Traits } & \multirow[b]{2}{*}{ Year } & \multicolumn{2}{|l|}{ Parents } & \multicolumn{4}{|l|}{ CSSLs } \\
\hline & & Nipponbare & 9311 & Average & Range & Kurtosis & Skewness \\
\hline \multirow[t]{4}{*}{$\mathrm{PV} / \mathrm{CP}$} & 2013 & $3,765.2 \pm 50.9$ & $3,372.4 \pm 14.0$ & 3573.4 & $3,003.0-4,074.0$ & 0.98 & -0.22 \\
\hline & 2014 & $3,243.6 \pm 44.5$ & $2,828.2 \pm 61.1$ & 3046.4 & $2,610.0-3,863.0$ & 2.4 & 0.47 \\
\hline & 2016 & $2,978.5 \pm 29.2$ & $2,197.9 \pm 22.6$ & 1993.6 & $920.0-2,459.0$ & 2.68 & -1.48 \\
\hline & 2017 & $3,372.3 \pm 38.7$ & $2,162.5 \pm 76.2$ & 1977.7 & $471.0-2,678.0$ & 2.26 & -1.28 \\
\hline \multirow[t]{4}{*}{$T V / C P$} & 2013 & $2,010.6 \pm 16.3$ & $1,950.0 \pm 13.2$ & 1965.2 & 1,719.0-2,189.0 & -0.38 & 0.13 \\
\hline & 2014 & $1,372.4 \pm 37.3$ & $1,294.3 \pm 23.5$ & 1490.5 & 1,232.0-1,992.0 & 1.09 & 0.92 \\
\hline & 2016 & $1,423.0 \pm 25.7$ & $1,290.5 \pm 24.1$ & 1231.4 & $403.0-1,901.0$ & 2.29 & -0.73 \\
\hline & 2017 & $2,373.3 \pm 124.2$ & $1,520 \pm 141.2$ & 1376.4 & $224.0-2,157.0$ & $1.29-0.80$ & -0.80 \\
\hline \multirow[t]{4}{*}{$\mathrm{FV} / \mathrm{CP}$} & 2013 & $3,395.5 \pm 48.1$ & $3,336.8 \pm 30.5$ & 3381.6 & $3,077.0-3,702.0$ & -0.44 & 0.12 \\
\hline & 2014 & $2,705.9 \pm 61.3$ & $2,657.1 \pm 58.2$ & 2859.2 & $2,477.0-3,280.0$ & -0.49 & 0.21 \\
\hline & 2016 & $2,961.4 \pm 36.2$ & $2,486.4 \pm 30.7$ & 2283.0 & $978.0-2,954.0$ & 4.29 & -1.55 \\
\hline & 2017 & $3,311.5 \pm 24.9$ & $2,685.3 \pm 149.3$ & 2550.4 & $668.0-3,562.0$ & 3.35 & -1.43 \\
\hline \multirow[t]{4}{*}{$\mathrm{BD} / \mathrm{CP}$} & 2013 & $1,755.4 \pm 28.6$ & $1,422.1 \pm 37.2$ & 1608.2 & 1,284.0-1,964.0 & 0.32 & 0.03 \\
\hline & 2014 & $1,873.6 \pm 65.4$ & $1,533.7 \pm 40.5$ & 1547.8 & $1,024.0-1,973.0$ & 0.06 & -0.25 \\
\hline & 2016 & $1,556.2 \pm 49.2$ & $907.3 \pm 26.5$ & 762.2 & $335.0-1116.0$ & 0.23 & -0.37 \\
\hline & 2017 & $999.2 \pm 51.4$ & $642.5 \pm 65.3$ & 601.3 & $247.0-911.0$ & -0.31 & -0.45 \\
\hline \multirow[t]{4}{*}{$\mathrm{SB} / \mathrm{CP}$} & 2013 & $-370.1 \pm 28.1$ & $-35.6 \pm 5.5$ & -191.7 & $-604.0-225.0$ & 0.17 & 0.14 \\
\hline & 2014 & $-541.3 \pm 59.1$ & $-170.5 \pm 30.6$ & -178.2 & $-659.0-489.0$ & -0.43 & 0.19 \\
\hline & 2016 & $-17.6 \pm 6.7$ & $288.8 \pm 12.3$ & 289.5 & $-171.0-639.0$ & 0.87 & -0.18 \\
\hline & 2017 & $-60.5 \pm 38.5$ & $523.0 \pm 73.4$ & 572.7 & 184.0-1060.0 & 1.05 & 0.13 \\
\hline \multirow[t]{4}{*}{$\mathrm{PeT} / \mathrm{min}$} & 2013 & $6.2 \pm 0.1$ & $5.9 \pm 0.1$ & 6 & $5.7-6.3$ & 1.79 & 0.58 \\
\hline & 2014 & $6.2 \pm 0.1$ & $6.1 \pm 0.0$ & 6.1 & $5.7-6.5$ & -0.51 & 0.12 \\
\hline & 2016 & $6.5 \pm 0.1$ & $6.2 \pm 0.1$ & 6.2 & $5.5-6.8$ & 0.78 & -0.10 \\
\hline & 2017 & $6.6 \pm 0.1$ & $6.6 \pm 0.2$ & 6.6 & 5.9-7.0 & 0.37 & -0.35 \\
\hline \multirow[t]{4}{*}{$\mathrm{PaT} /{ }^{\circ} \mathrm{C}$} & 2013 & $74.7 \pm 0.9$ & $86.3 \pm 3.1$ & 81.9 & $73.6-87.1$ & -1.31 & -0.69 \\
\hline & 2014 & $76.7 \pm 0.5$ & $74.4 \pm 0.6$ & 77.9 & $72.0-88.6$ & -1.48 & 0.63 \\
\hline & 2016 & $76.0 \pm 0.3$ & $74.4 \pm 1.2$ & 76.4 & $72.0-92.6$ & 4.74 & 2.48 \\
\hline & 2017 & $77.1 \pm 0.4$ & $76.8 \pm 0.4$ & 79.3 & 729.-94.9 & 0.92 & 1.65 \\
\hline
\end{tabular}



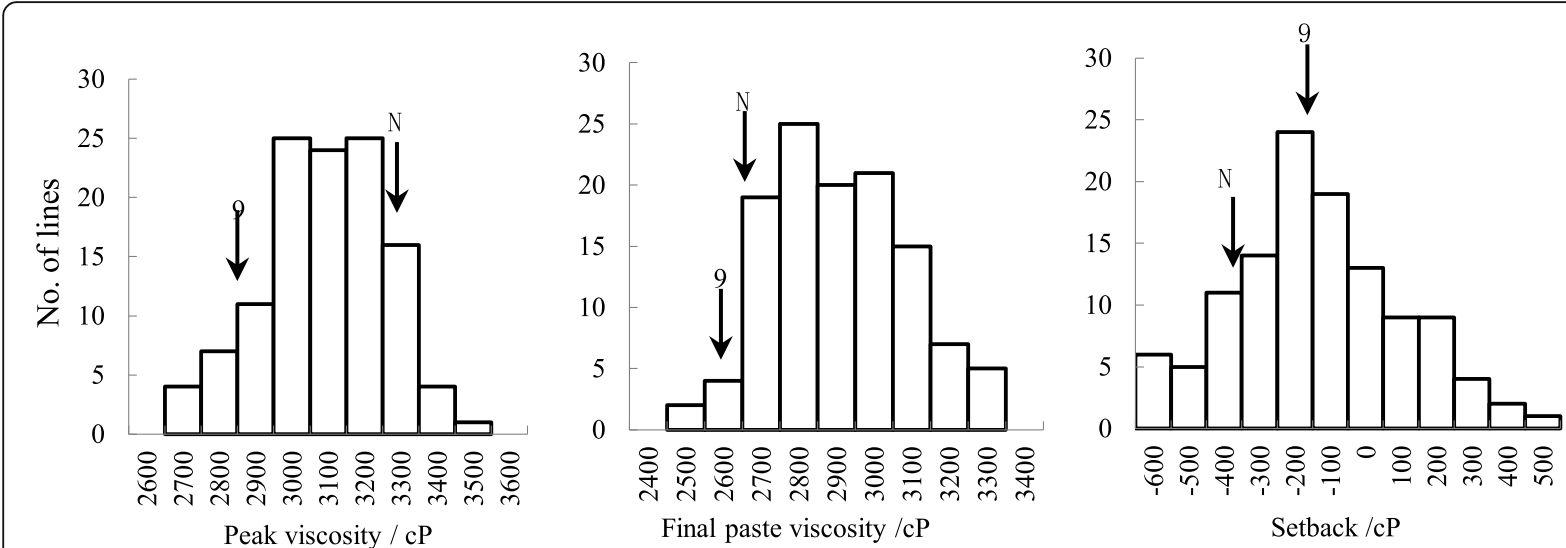

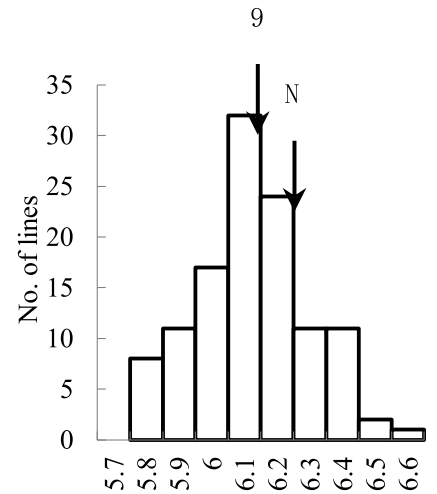

Peak time / $\min$

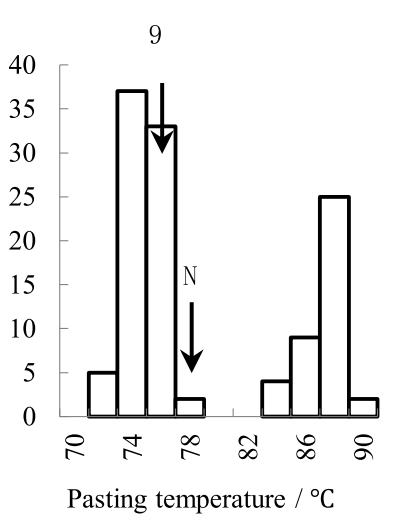

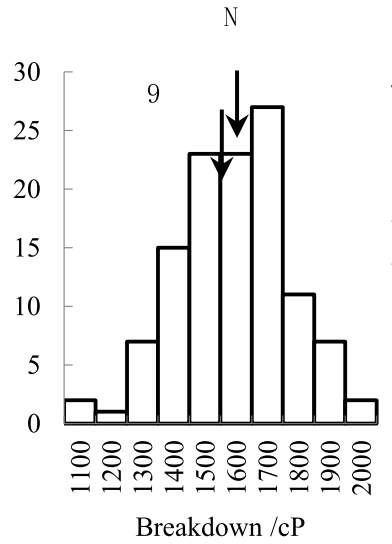

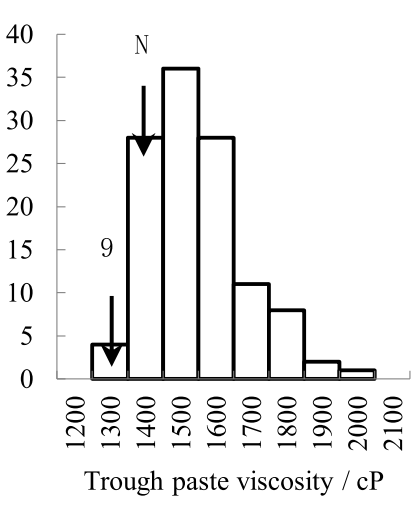

Fig. 1 Distribution of starch rapid visco analyzer profile characteristics in the Nipponbare/9311 chromosome segment substitution line population in 2014. Note: The mean values for 9311 and Nipponbare are indicated by arrows labeled 9 and N, respectively

(Table 1). In addition, the phenotypic values for all starch RVA profiles showed bidirectional ultra-parental genetic types in the CSSL population, consistent with polygenic control of those quantitative traits.

Correlation analysis using average values for each year as a variable revealed that PV was positively correlated with trough viscosity (TV), BD, and FV, but negatively correlated with SB and PaT. TV was positively correlated with FV and PeT. FV was positively correlated with PeT and $\mathrm{PaT}$ but negatively correlated with $\mathrm{SB}$. BD was negatively correlated with all other RVA parameters. SB was positively correlated with $\mathrm{PeT}$ and $\mathrm{PaT}$. These correlations were highly significant $(P<0.01)$. Over four years, the relationship between $\mathrm{SB}$ and TV or FV changed (Supplementary Tables 1, 2, 3). SB was positively correlated with FV except in 2013. TV was positively correlated with SB in 2016 and 2017.

\section{Microclimate analysis}

Every year, 9311 and Nipponbare bloomed on July 20th and August 25th, respectively. The heading time of CSSLs ranged from August 15th to the 25th. Although the maximum temperature of July and August was more than $40{ }^{\circ} \mathrm{C}$ in 2017 , CSSLs and parents showed normal seed sets across the four years. The mean daily maximum temperature during the recorded periods ranged from 28.7 to $34.3{ }^{\circ} \mathrm{C}$ over the four years of this study. The daily average temperatures during observational periods in 2013, 2016, and 2017 were about $28.0^{\circ} \mathrm{C}$, higher than the average temperature of $25.1{ }^{\circ} \mathrm{C}$ measured in 2014 (Fig. 2). The average daily maximum temperatures showed the same tendency as the daily average temperatures over all four years. The highest recorded temperature of the four years was in 2017. The relative humidity $(\mathrm{RH})$ varied extensively over four years independently of temperature. Average daily $\mathrm{RH}$ during the observational periods was higher in 2014 and 2017 at about $91 \%$ lower than those in 2013 and 2016 at 80.5 and $82.5 \%$.

\section{QTL analysis}

To elucidate the effects of environmental factors on starch viscosity among CSSLs, we mapped QTLs that influence starch RVA profile characteristics each year. In 


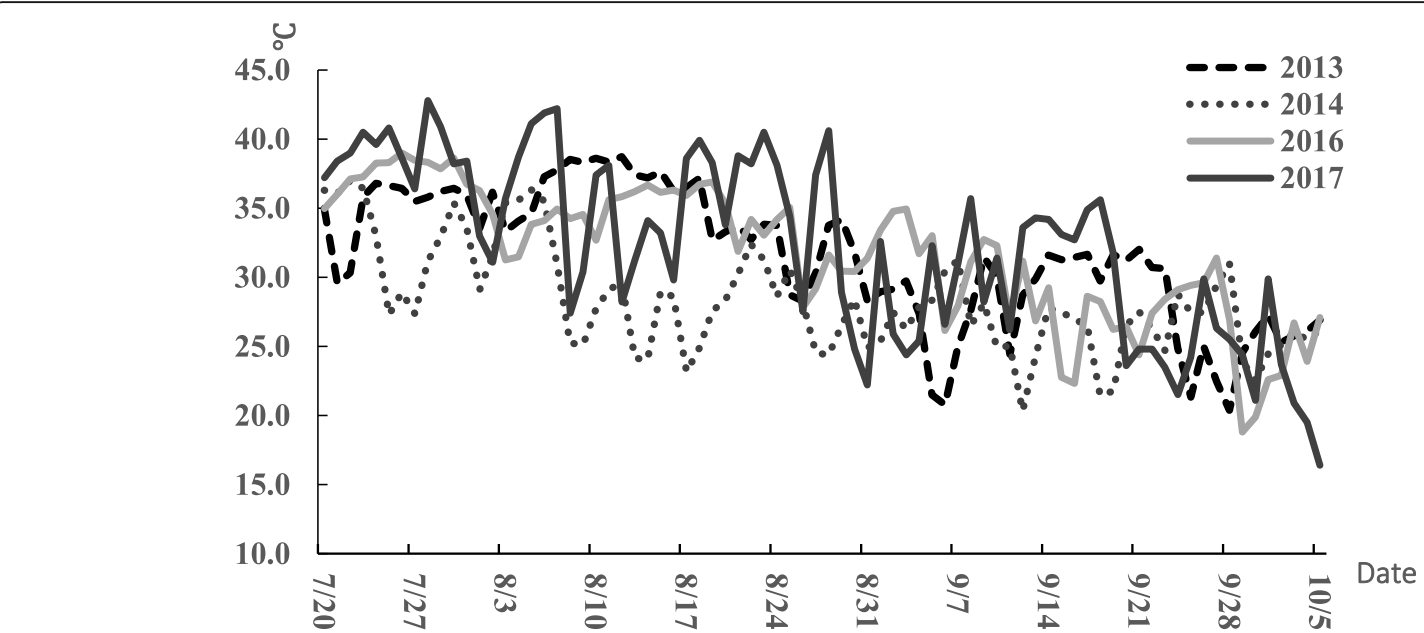

Fig. 2 Daily maximum temperature recorded during the observational periods over the four years of this study

total, 310 QTLs were detected for seven RVA characteristics. Of them, 44 QTLs were related to PV, 42 to TV, and 43 to FV; 43, 49, 34, and 55 QTLs were identified for $\mathrm{BD}, \mathrm{SB}, \mathrm{PeT}$, and $\mathrm{PaT}$, respectively.

22 loci for PV, 15 for TV, 25 for FV, and 11 for BD were stably detected across multiple years. 28,8 , and 27 QTLs were mapped in different years for $\mathrm{SB}, \mathrm{PeT}$ and PaT, respectively. Totally, 81 QTLs were mapped to the same genomic region across two years, and 50 QTLs were identified across three years. Five stable QTLs controlling TV, FV and PaT were identified across all four years (Table 2). The remaining 174 QTLs appeared in only one year. These results suggested that, although most traits showed large variation across the years, several QTLs related to starch RVA profile characters were robust enough to be consistently identified each year. For example, we repeatedly detected a QTL around 16.9-19 Mb of chromosome 2 that affects both FV and TV each year. Similarly, $q \mathrm{PaT7}$ and $q T V 7$ located near marker RM432 on chromosome 7 and $q F V 9$ located near RM219 on chromosome 9 were also mapped every year. A QTL near marker RM3827 on chromosome 6 for SB and a QTL near marker RM1812 on chromosome 11 for BD have not been previously described to be associated with these traits at the genomic locations (Table 3). Six QTLs were not affected by environmental factors in our research, and they are likely to substantially contribute to the RVA characteristics of rice starch.

Several of the QTLs detected here exhibited pleiotropic effects, as they influenced multiple starch RVA profile characteristics. In total, 26 genomic intervals corresponding to 55 QTLs associated with starch RVA profiles were identified in at least three years. Of those, 13 chromosomal intervals showed pleiotropy by affecting more than one starch RVA profile characteristic (Table 3). In particular, the region near marker RM219 on chromosome 9 contained one QTL associated with all starch RVA profile characteristics with the exception of TV. Moreover, the interval between markers RM469 and RM587 on chromosome 6 was associated with five parameters, while the interval near marker RM3795 on

Table 2 Quantitative trait locus (QTL) analysis for properties of the starch rapid visco analyzer profile

\begin{tabular}{lccccc}
\hline Trait & No. of QTLs & & & \\
\cline { 2 - 6 } & Mapped over two years & Mapped over three years & Mapped over four years & Total mapped QTLs & Repeatedly detected QTL (\%) \\
\hline $\mathrm{PV} / \mathrm{CP}$ & 18 & 4 & 0 & 44 & 50 \\
$\mathrm{TV} / \mathrm{CP}$ & 10 & 3 & 3 & 42 & 35.7 \\
$\mathrm{FV} / \mathrm{CP}$ & 13 & 8 & 2 & 43 & 58.1 \\
$\mathrm{BD} / \mathrm{CP}$ & 3 & 13 & 0 & 43 & 25.6 \\
$\mathrm{SB} / \mathrm{CP}$ & 15 & 3 & 0 & 49 & 57.1 \\
$\mathrm{PeT} / \mathrm{min}$ & 5 & 9 & 0 & 34 & 23.5 \\
$\mathrm{PaT} /{ }^{\circ} \mathrm{C}$ & 17 & 50 & 1 & 55 & 49.1 \\
Total & 81 & & 6 & 310 & 43.9
\end{tabular}


Table 3 Stable rapid visco analyzer (RVA) quantitative trait loci (QTLs) detected more than three times

\begin{tabular}{|c|c|c|c|c|}
\hline Chromosome & Marker & $\begin{array}{l}\text { Interval } \\
(\mathrm{Mb})\end{array}$ & Traits & Other RVA QTL co-located \\
\hline 1 & RM488 & $25.75-27$ & SB & qBDV1 [18]; aBDV1 [24] \\
\hline 1 & RM3143 & $28.05-29$ & $\mathrm{BD}$ & aPKV1 [24] \\
\hline 2 & RM5390 & $10.15-11.11$ & $\mathrm{PaT}, \mathrm{TV}$ & qCPV2, aSBV2 [34]; qBDV2a, aCSV2a, qTD2 [23] \\
\hline 2 & RM3795 & $16.9-20.95$ & $\mathrm{FV}^{\mathrm{a}}, \mathrm{TV}^{\mathrm{a}}, \mathrm{SB}, \mathrm{PaT}$ & aPKV2, aCPV2, aSBV2 [34]; qPeT2 [15] \\
\hline 2 & RM191 & $25.75-29.3$ & $\mathrm{FV}$ & qBDV2 [35]; qHPV2 [34]; qPaT [27] \\
\hline 2 & RM1342 & $28.65-29.3$ & PV, PaT & qHPV2 [35]; qPKV2 [18] \\
\hline 4 & RM518 & $1.1-3.24$ & SB & qPKV4 [27] \\
\hline 4 & $\begin{array}{l}\text { RM6748- } \\
\text { RM5473 }\end{array}$ & $30.45-32.65$ & SB & qPT4 [35]; qASV [36] \\
\hline 5 & RM289 & $7.13-8.55$ & $\mathrm{BD}, \mathrm{PaT}, \mathrm{SB}, \mathrm{FV}$ & qSBV5, qCSV5 [34]; qHPV5 [35]; qPV5, qHPV5, qPeT5 [15] \\
\hline 5 & RM178 & $24.59-26.37$ & $\mathrm{BD}, \mathrm{FV}, \mathrm{SB}, \mathrm{PaT}$ & qPKV5, aHKV5 [22] \\
\hline 6 & RM469-RM587 & $0.56-2.86$ & $\mathrm{SB}, \mathrm{PV}, \mathrm{BD}, \mathrm{PeT}, \mathrm{PaT}$ & $\begin{array}{l}\text { qPeT6 [3]; qTPV6, qFPV6, qBDV6, qSBV6, qPKT6 [21]; qHPV6-1, } \\
\text { qCPV6, qCS6, qSBV6 [7]; qPKV6, qBDV6, qSBV6, qPeT6 [24]; } \\
\text { qBD6, aSB6 [1]; qTV6, qCPV6, qSBV6, qPeT6, qPKV6 [25] }\end{array}$ \\
\hline 6 & RM527 & $9.31-10.98$ & $\mathrm{SB}, \mathrm{FV}$ & qPeT6 [24] \\
\hline 6 & RM3827 & $22.55-23.8$ & SB & \\
\hline 6 & RM3628 & $23.8-24.8$ & PV & qHPV6 [1] \\
\hline 6 & RM412 & $30.85-31.59$ & SB & qBDV6, qCSV6, qCPV6, qHPV6, qSBV6 [18] \\
\hline 7 & RM542 & $12.41-14.5$ & PeT & qGT7 [23] \\
\hline 7 & RM432 & $18.6-20.55$ & $\mathrm{PaT}^{\mathrm{a}}, \mathrm{TVa}$ & qCPV7 [18]; qHPV7, aBDV7, aCPV7 [24]; qGT7-1 [23]; qPaT-7, qPeT-7 [25] \\
\hline 8 & RM5485 & $23.32-25.4$ & $\mathrm{FV}$ & qBDV8, qPeT8 [34]; qPKV8 [24]; qPKV8, aHPV8, aHPV6 [3] \\
\hline 9 & RM219 & $7.39-9.11$ & $\begin{array}{l}\mathrm{BD}, \mathrm{PV}, \mathrm{SB}, \mathrm{PaT}, \mathrm{PeT} \\
\mathrm{FV}^{\mathrm{a}}\end{array}$ & qPaT9 [15]; qPKV9,aBDV9-b [25] \\
\hline 9 & RM566 & $14.63-16.15$ & PV, PeT & qPKV10 [34]; qHPV9, qHPV9 [1]; qCPV9, qCS9 [15] \\
\hline 10 & $10-1.63$ & $0.8-2.12$ & $\mathrm{BD}, \mathrm{FV}, \mathrm{SB}, \mathrm{TV}$ & qCPV10, aSBV10, aCSV10 [3]; qBDV10, qPeT10 [37] \\
\hline 10 & 10-9.12 & $8.76-9.57$ & $\mathrm{TV}^{\mathrm{a}}, \mathrm{FV}, \mathrm{BD}$ & aPKV10, aHPV10, qCPV10, aSBV10, aPeT10, qCSV10 [3]; qSB10 [27] \\
\hline 10 & RM1375 & 15.89-18.04 & $\mathrm{BD}$ & $\begin{array}{l}\text { qCPV10; qSBV10; qPeT10, aHPV10, aPKV10 [3]; qPET10, qPAT10, qPKV10, aPKV10 } \\
\text { [34] }\end{array}$ \\
\hline 11 & RM1812 & $1.35-2.61$ & $\mathrm{BD}$ & qBDV11, qPKV11,qPaT11 [25] \\
\hline 12 & RM1261 & $15.52-18.07$ & $\mathrm{FV}$ & qGT12 [23]; qPaT12 [15]; qBDV12, qPaT12 [25] \\
\hline 12 & RM1227 & $27.4-27.6$ & $\mathrm{FV}, \mathrm{SB}$ & qGT12 [23] \\
\hline
\end{tabular}

The QTL was detected in each of the four years of the study

chromosome 2, markers RM289 and RM178 on chromosome 5, and marker 10-1.63 on chromosome 10 were each associated with four RVA parameters.

Several reports have described QTLs affecting rice starch RVA profiles [18, 21-23, 34]. The 26 intervals detected more than three times were compared with previously mapped QTL. With the exception of two intervals, all other intervals co-located with known QTLs associated with starch RVA profiles from different mapping populations and different environments (Table 3). Specifically, a number of certain hot spots were identified, such as the region between markers RM469 and RM587 on chromosome 6 and the region around marker RM1375 on chromosome 10. In the genomic interval from markers RM469 to RM587 on chromosome 6, we mapped QTLs for SB, PV, BD, PeT, and PaT, as the previous studies did for the traits PeT, TV, FV, BD, and SB [1, 3, 7, 21, 24]. qBD10, located near RM1375 (15.9-18 Mb) on chromosome 10, overlapped with the mapping interval described for certain starch RVA profile QTLs related to FV, SB, PeT, TV, PaT, and PV [3, 24, 34].

We noticed that several of our stable QTL hotspots co-located with some known SSRGs, such as Wx, SSIII1, SSIV-2, and SBE3 (Table 4). Besides the QTL in the interval $0.56-2.86 \mathrm{Mb}$ on chromosome 6 , which colocated with $W x$, the region spanning $3.24-5.38 \mathrm{Mb}$ and 30.45-32.65 Mb on chromosome 4, 24.59-26.37 Mb on chromosome 5, and 16.9-20.95 $\mathrm{Mb}$ on chromosome 2 contained PUL, SSIII-1, SSIV-2, and SBE3, respectively. The loci ADPlar (Adenosine diphosphate glucose 
pyrophosphorylase large subunit 1), ADPsma (Adenosine diphosphate glucose pyrophosphorylase small subunit), SSII-1, and ISA were near our starch RVA QTLs. In the case of ADPlar, ADPiso (Adenosine diphosphate glucose pyrophosphorylase large subunit 2), GBSSII, SSII-2, SSIII2, SSIV-1, and PUL, the nearby or overlapping QTLs were identified in only one or two years, suggesting that the underlying QTLs are easily influenced by the environment. No QTLs associated with starch RVA traits was detected in regions that overlap with the genomic location of SSI, SSII-3, SBE1, or SBE4. The region between markers RM6748 and RM5473 on chromosome 4 contained a QTL for SB and overlapped with the genomic location of SSIII-2, which is related to SB. SSIII-2 is a critical gene controlling Pat and PeT [6]. The effect of this region on Pat and $\mathrm{PeT}$ was not tested in our research.

The QTL hotspots near SSII-1 and SSII-3 were previously reported to have major effects on $\mathrm{PT}$ and minor effects on gel consistency (GC), AC, PV, CPV, BD, and SB [2]. Here, we found that the QTL near SSII-1 is associated with BD. A QTL hotspot near SBE3 on chromosome 2 was also reported, in which four major QTLs associated with HPV, consistency viscosity, viscosity at $95{ }^{\circ} \mathrm{C}$, and BD over two years [27]. We also detected QTL hotspots that co-located with SBE3 and are associated with FV, TV, SB, and PaT.

\section{Sequence alignment of SSRGs between parents 9311 and Nipponbare}

The stable QTL hotspots were co-located with several known SSRGs. To explore the potential sequence variation between the two parental cultivars at these SSRGs, we aligned the nucleotide and predicted protein sequences from 9311 and Nipponbare, which revealed limited allelic diversity between the two parents, with some exceptions (Table 4). For example, the parental alleles for SSI and SSII-3 were identical in sequence in 9311 and Nipponbare, possibly explaining why we failed to detect a QTL overlapping with either of these intervals in this study. Likewise, we detected no QTL near SBE1 and $S B E 4$, whose sequence identity between the two parents was 99.71 and $99.87 \%$, respectively.

The two parents also shared the same allele at ADPlar and $S S I V-2$, whose genomic coordinates did not overlap with any of our QTL intervals. However, our QTLs related to RVA profile parameters overlapped with or in close proximity to 12 SSRGs. Although the gene sequences of $W x$ are identical between 9311 and Nipponbare, we identified QTLs associated with multiple RVA traits in the $W x$ region. In addition, the nucleotide sequences and encoded protein sequences for ADPsma, SSII-1, SSII-2, SSIII-1, SSIII-2, SSIV-1, SBE3, ISA, and $P U L$ were different between the two parents, suggesting that sequence polymorphisms at these loci contribute to the observed QTLs controlling RVA profile characteristics.

\section{Discussion}

Recent studies have confirmed that changes in the environment largely affect rice starch PV, TV, and FV, while $\mathrm{AC}$ and other starch RVA parameters are mainly influenced by genotypes $[1,11]$. In this study, starch RVA profiles of both parents and CSSLs varied greatly across four years, especially in 2016 and 2017. One possible reason is the high temperature of the grain filling stage during the summer of these years [38-40]. High temperature during flowering will make the spikelet sterile and reduce the seed set. Air temperature during grain filling significantly affects grain composition, as well as starch structure and properties [41, 42]. High temperatures result in lower $\mathrm{AC}$, poor ECQs, and higher pasting properties by decreasing the activity of starch synthesis enzymes. Under such conditions, rice starch contained fewer long-chain amylose and short-chain amylopectin, but more intermediate- and long- chain amylopectin than under normal conditions, as well as higher crystallinity and gelatinization properties [42].

We observed that PV is positively correlated with TV, $\mathrm{BD}$, and FV but negatively correlated with $\mathrm{SB}$ and PaT. Similar correlations were previously reported $[1,3,34]$. We also established that PV, FV, TV, BD, PaT, and PeT show consistent cross-correlations regardless of their great variations across the years, indicating that these traits are mainly affected by genotypic variation. There were main effect loci that controlled these starch RVA profile characteristics. The interaction between genotype and environment exhibited strongly for SB, as the correlation coefficients between SB and other parameters (except PV) differed for each year.

The confounding effects of the environment and the major effect gene $W x$ make it difficult to identify more minor QTLs for starch RVA profiles. Only a few genes and QTLs related to starch RVA profiles have been cloned, such as qAC2, qGC6, ALK, Chalk5, and Dul (Dull) [6]. In this study, we specifically selected two cultivars with the same $W x$ allele, $W x-b$, to generate CSSLs as the mapping population [22]. SSII-3, the major gene controlling GT, showed no differences in its genomic sequence and only a 2-amino acid difference in its protein sequence between 9311 and Nipponbare. We therefore hypothesize that 9311 and Nipponbare carry the same allele at SSII-3. New QTLs that exert minor effects on starch RVA profiles and GT could be identified without the influence of major $W x$ and SSII-3. Zhang developed a CSSL population consisting of 38 lines with the same parents selected here. Following the same mapping strategy, 10 stable QTLs for RVA properties and two minor 
Table 4 Co-location of known SSRGs with the starch rapid visco analyzer (RVA) quantitative trait loci (QTLs) detected in this study

\begin{tabular}{|c|c|c|c|c|c|c|c|}
\hline Gene & Acc. number & $\begin{array}{l}\text { Position } \\
\text { (Mb) }\end{array}$ & $\begin{array}{l}\text { Traits associated with QTL in } \\
\text { this study }\end{array}$ & $\begin{array}{l}\text { QTL interval } \\
(\mathrm{Mb})\end{array}$ & $\begin{array}{l}\text { Identity between } 9311 \text { and } \\
\text { Nipponbare (\%) }\end{array}$ & $\begin{array}{l}\text { No. of } \\
\text { Gap }\end{array}$ & $\begin{array}{l}\text { No. of } \\
\text { different aa }\end{array}$ \\
\hline ADPlar & $\begin{array}{l}\text { LOC_ } \\
\text { Os05g50380 }\end{array}$ & $\begin{array}{l}\text { Chr.5, } \\
28.87\end{array}$ & $\mathrm{BD}, \mathrm{FV}$ & $27.95-28.85$ & 99.82 & 10 & 0 \\
\hline ADPiso & $\begin{array}{l}\text { LOC_ } \\
\text { Os01g44220 }\end{array}$ & $\begin{array}{l}\text { Chr.1, } \\
25.35\end{array}$ & $\underline{\mathrm{BD}, \mathrm{PT}, \mathrm{PeT}}$ & $23.85-25.75$ & 99.78 & 4 & 0 \\
\hline ADPsma & $\begin{array}{l}\text { LOC_ } \\
\text { Os09g12660 }\end{array}$ & Chr.9, 7.24 & $\mathrm{BD}, \mathrm{PV}, \mathrm{SB}, \mathrm{PaT}, \mathrm{PeT}, \mathrm{FV}^{\mathrm{a}}$ & $7.39-9.11$ & 99.75 & 9 & 5 \\
\hline $\begin{array}{l}\text { GBSSI } \\
(W x)\end{array}$ & $\begin{array}{l}\text { LOC_ } \\
\text { Os06g } 04200\end{array}$ & Chr.6, 1.70 & $\mathrm{SB}, \mathrm{PV}, \mathrm{BD}, \mathrm{PeT}, \mathrm{PaT}$ & $0.56-2.86$ & 100 & 0 & 0 \\
\hline SSI & $\begin{array}{l}\text { LOC_ } \\
\text { Os06g } 06560\end{array}$ & Chr.6, 3.08 & - & - & 99.94 & 7 & 0 \\
\hline SSII-1 & $\begin{array}{l}\text { LOC_ } \\
\text { Os10g30156 }\end{array}$ & $\begin{array}{l}\text { Chr.10, } \\
15.67\end{array}$ & BD98.06-100 & 15.89-18.04 & 99.64 & 8 & 4 \\
\hline$S S I I-2$ & $\begin{array}{l}\mathrm{LOC}_{-} \\
\text {Os02g51070 }\end{array}$ & $\begin{array}{l}\text { Chr.2, } \\
31.23\end{array}$ & $\underline{\mathrm{PK}, \mathrm{PK}}$ & $30.45-34.75$ & 99.66 & 3 & 4 \\
\hline SSII-3 & $\begin{array}{l}\text { LOC_ } \\
\text { Os06g12450 }\end{array}$ & Chr.6, 6.75 & - & - & 100 & 0 & 0 \\
\hline SSIII-1 & $\begin{array}{l}\text { LOC_ } \\
\text { Os04g53310 }\end{array}$ & $\begin{array}{l}\text { Chr.4, } \\
31.76\end{array}$ & SB & $30.45-32.65$ & 99.81 & 24 & 4 \\
\hline SSIII-2 & $\begin{array}{l}\text { LOC_ } \\
\text { Os08g09230 }\end{array}$ & Chr.8, 5.35 & $\underline{\mathrm{BD}, \mathrm{FV}, \mathrm{PK}, \mathrm{TV}}$ & $4.75-6.28$ & 99.61 & 9 & 57 \\
\hline SSIV-1 & $\begin{array}{l}\mathrm{LOC}_{-} \\
\text {Os01g52250 }\end{array}$ & $\begin{array}{l}\text { Chr.1, } \\
30.04\end{array}$ & $\underline{\mathrm{PK}, \mathrm{ST}, \mathrm{TV}}$ & $29.75-35.1$ & 98.06 & 16 & 152 \\
\hline SSIV-2 & $\begin{array}{l}\text { LOC_ } \\
\text { Os05g45720 }\end{array}$ & $\begin{array}{l}\text { Chr.5, } \\
26.48\end{array}$ & $\mathrm{BD}, \mathrm{FV}, \mathrm{SB}, \mathrm{PaT}$ & $24.59-26.37$ & 99.7 & 10 & 0 \\
\hline SBE1 & $\begin{array}{l}\text { LOC_ } \\
\text { Os06g } 26234\end{array}$ & $\begin{array}{l}\text { Chr.6, } \\
15.33\end{array}$ & - & - & 99.71 & 33 & 65 \\
\hline SBE3 & $\begin{array}{l}\text { LOC_ } \\
\text { Os02g32660 }\end{array}$ & $\begin{array}{l}\text { Chr.2, } \\
19.36\end{array}$ & $\mathrm{FV}^{\mathrm{a}}, \mathrm{TV}^{\mathrm{a}}, \mathrm{SB}, \mathrm{PaT}$ & $16.9-20.95$ & 99.69 & 77 & 2 \\
\hline SBE4 & $\begin{array}{l}\text { LOC_ } \\
\text { Os04g33460 }\end{array}$ & $\begin{array}{l}\text { Chr.4, } \\
20.12\end{array}$ & - & - & 99.87 & 15 & 21 \\
\hline ISA & $\begin{array}{l}\text { LOC_ } \\
\text { Os08g40930 }\end{array}$ & $\begin{array}{l}\text { Chr.8, } \\
25.89\end{array}$ & $\mathrm{FV}$ & $23.32-25.4$ & 99.59 & 17 & 2 \\
\hline PUL & Os04G0164900 & Chr. $4,4.40$ & $\underline{\mathrm{BD}, \mathrm{PeT}, \mathrm{ST}}$ & $3.24-5.38$ & 74.69 & 402 & 78 \\
\hline
\end{tabular}

${ }^{a}$ The QTL was detected in each of the four years

_ The QTL was detected only once or twice

stable QTLs for GT were identified over two years and in two environments [22, 23].

The QTLs for the RVA properties they mapped also displayed pleiotropy but didn't map to the same or similar chromosomal regions with our QTLs, which might not be surprising in considering that the two sets of CSSL lines harbored different chromosome segments from Nipponbare. Liu et al. (2011) also performed an extensive QTL mapping analysis for 16 rice quality traits across eight environments using a set of CSSLs developed from two rice varieties with similar $\mathrm{AC}$, and detected 10 stable RVA profiles cross four environments [19]. However, the number of molecular markers was limited, raising the possibility of missing small introgression segments and thus lowering the accuracy of QTL detection. With more lines and smaller substitution segments, our CSSLs are adequate to identify minor QTLs for starch RVA profiles and GT without the effect of SSII-3 and Wx.

In our research, although 310 QTLs were detected for seven paste viscosity properties for rice starch RVA profile traits, only 136 QTLs were mapped repeatedly. These results indicated that the RVA profile characteristics are largely affected by the environment, consistent with previous reports $[18,19]$. The novel loci we detected for all starch RVA parameters, and in particular the six QTLs we identified every year, are important and require further validation. In addition, we propose that new and stable QTLs, such as $q S B 6$ and $q B D 11$, which had not been previously reported, may be useful for research on marker-assisted selection of ECQs.

Based on our mapping, the clustering of multiple QTLs controlling RVA traits indicate that pleiotropic effects and QTL hotspots are key factors affecting starch 
RVA traits in rice. Similar observations of pleiotropy were reported previously (http://qtaro.abr.affrc.go.jp/, [3, 24]). QTLs with high correlations are often grouped in the same or adjacent marker intervals on a chromosome [43]. Starch RVA traits showed significant correlations that confirmed the linkage or pleiotropy of the corresponding loci.

Overlap between the intervals of our mapped QTLs and known SSRGs was common in this research (Table 4). Most known SSRGs showed different alleles between the parents, 9311, and Nipponbare. This result suggested that alleles of SSRGs contributed to our QTLs controlling RVA profile characteristics under the same major gene $W x$, which should be taken into consideration in rice quality breeding. The effect of SSRG allele combinations on starch quality and ECQs should be investigated more in the future. We failed to identify QTLs associated with RVA profiles near some SSRGs, such as SSII-3 and SSI, likely due to the lack of allele polymorphism between the two parents. The 5 untranslated region (UTR), genomic and protein sequences of $W x$ are identical between 9311 and Nipponbare, although we noted several RVA traits associated with the $W x$ genomic region. $\mathrm{Xu}$ also reported several SNPs close to $W x$ that were significantly associated with RVA parameters in subpopulations with the same Wx allele [15]. These loci will be high-priority candidates for future characterization.

The highly consistent genotypes between SSRGs and QTLs reported here confirmed the accuracy of our mapping. The stable or newly developed QTLs located in the region that no known SSRGs in are meaningful to identify new genes controlling starch RVA profiles in the future, which will facilitate further research into the genetic mechanism regulating RVA profiles and ECQ of rice.

\section{Conclusions}

We mapped QTLs associated with starch viscosity profile, one of the most important factors contributing to ECQs in rice. The effect of major locus $W x$ could be eliminated by generating CSSLs developed from 9311 and Nipponbare with the same $W x-b$ allele, which allowed the identification of new QTLs associated with RVA profile traits. We analyzed the genetic basis of variation in RVA profile over four years, and identified 136 repeated QTLs. Among them, 6 stable QTLs were detected every year, which are therefore likely to be very important for the RVA characteristics. In addition, we uncovered 13 intervals detected for 3 of the four years that showed pleiotropy with respect to controlling two to six starch RVA profile properties simultaneously. Finally, we highlighted four intervals, such as the interval between markers RM469 and RM587 on chromosome 6, that are associated with more than four RVA parameters for a given year. Pleiotropic effects and QTL hotspots appear to be key factors affecting starch RVA traits in rice. There was high consistency between allelic diversity at known SSRGs and the QTLs reported here. Future research will explore these stable QTLs and hotspots in more detail.

\section{Methods}

\section{Plant materials and field planting}

An advanced backcross population was developed by our lab using the indica variety '9311' (recipient) and the japonica variety 'Nipponbare' (donor). 9311 was obtained from its breeder, Yangzhou Institute of Agricultural Sciences, Jiangsu Academy of Agricultural Sciences. Nipponbare was obtained from Jiangsu Provincial Platform for Conservation and Utilization of Agricultural Germplasm.

The backcrossed population consisted of $119 \mathrm{BC}_{4} \mathrm{~F}_{2}$ lines. Backcrossing and simple sequence repeat marker selection were performed as described in detail by $\mathrm{Zhu}$ and Zhao [20, 31]. Each introgression line in the population contained one to seven segments originating from Nipponbare. The 119 lines contained 318 substituted segments with an average of two to seven segments per line and covered $84.0 \%$ of the whole rice genome [31].

The CSSLs and two parents were planted in fields at the Jiangsu Academy of Agricultural Sciences $\left(32^{\circ} 02^{\prime} \mathrm{N}\right.$, $118^{\circ} 52^{\prime} \mathrm{E}$; elev. $10 \mathrm{~m}$ ) in Nanjing in 2013, 2014, 2016, and 2017. Each year, all seeds were planted within two blocks on May 15th and transplanted on June 20th. Each line was planted in three rows with a row-to-row distance of $30 \mathrm{~cm}$ and plant-to-plant distance of $13.3 \mathrm{~cm}$. The seeds of five plants were sampled from each CSSL line and dried naturally. Milled rice was grinded into powder and then passed through a 100-mesh sieve. After drying at $4{ }^{\circ} \mathrm{C}$ in an oven, each powder sample was balanced for 2 days at room temperature and kept at $4{ }^{\circ} \mathrm{C}$ for 3 months. Then, paste viscosity was measured as described below. For each line, five samples were used to determine the starch RVA profile.

\section{Microclimate}

The climate parameters at the field site (air temperature, $\mathrm{RH}$ ) were measured using a data logger (Thermo Recorder TR-72U, T \& D Corp, Japan). The sensor was placed at a height of $170 \mathrm{~cm}$; air temperature and $\mathrm{RH}$ were collected every $10 \mathrm{~min}$. The climate parameters were collected from the beginning of flowering to full maturity each year.

\section{Starch RVA profile}

Starch paste viscosity was measured with a Rapid Visco Analyser (Tecmaster, Perten, Sweden) according to the American Association of Cereal Chemists Standard 
Method (AACC 61 - 02) with TCW software 3 (Thermal Cycle for Windows) [44]. $3 \mathrm{~g}$ rice flour (accounting for $12 \%$ moisture basis) with $25 \mathrm{~mL}$ distilled water were used. The heat profile was set as follows: (1) the temperature was held at $50{ }^{\circ} \mathrm{C}$ for $1 \mathrm{~min}$; (2) the temperature was linearly ramped up to $95{ }^{\circ} \mathrm{C}$ over $3.75 \mathrm{~min}$; (3) the temperature was held at $95{ }^{\circ} \mathrm{C}$ over $2.5 \mathrm{~min}$; (4) the temperature was ramped down linearly to $50{ }^{\circ} \mathrm{C}$ for $3.75 \mathrm{~min}$; (5) the temperature was held at $50{ }^{\circ} \mathrm{C}$ for $1.4 \mathrm{~min}$. The RVA paddle speed was set to $960 \mathrm{rpm}$ for the first $10 \mathrm{~s}$ of the test, after which the speed was $160 \mathrm{rpm}$.

Starch paste viscosity characteristics are characterized by five parameters : PV, TV, FV, PeT, and PaT. BD and $\mathrm{SB}$ were calculated as: $\mathrm{BD}=\mathrm{PV}-\mathrm{TV}$, and $\mathrm{SB}=\mathrm{FV}-\mathrm{PV}$ [36]. Correlations between the RVA parameters for each year were analyzed by IBM SPSS Statistics v22.

The AAC was determined using the iodine staining method described in the European Standard EN ISO 6647-2-2015. The absorbance of the solution was measured at $620 \mathrm{~nm}$ against the blank solution using a continuous flow analyzer (Seal Analytical AA3, GER). The AAC was calculated using a standard curve made from four rice samples with known AAC.

\section{QTL mapping}

Genotype data for 250 polymorphic loci, including 211 simple sequence repeat and 39 sequence tag site markers, were used for QTL detection. Molecular linkages were established using composite interval mapping with version 3.3 of QTL IciMapping software [45].

QTL were detected according to the method described by Eshed and Zamir [46]. The significance of each QTL was determined by comparing the mean RVA profile values of a CSSL line with the recipient parent 9311 using analysis of variance and Dunnett's test. A QTL was considered as present when a CSSL line exhibited a significant difference compared to 9311 with corresponding probability value $P<0.05$. If more than three CSSLs showed differences, then the QTL was estimated as being located within the chromosomal region shared by those CSSLs [22]. QTL nomenclature followed as that of McCouch et al. [47].

\section{Sequence alignment of SSRGs between parents 9311 and Nipponbare}

The genomic and predicted protein sequences of known SSRGs that co-locate with starch RVA QTLs were downloaded from online resources, as 9311 and Nipponbare genomes are sequenced. Nipponbare sequences were downloaded from Gramene (http://www.gramene. org/). The genomic sequences for 9311 were obtained from the Rice Genome Project (Beijing Genomics Institute) [32]. The predicted protein sequences were obtained from Gramene or NCBI (https://www.ncbi.nlm. nih.gov/). Sequence alignment was performed with DNAMAN (version 6).

\section{Abbreviations}

ECQs: Eating and cooking qualities; RVA: Rapid Visco Analyzer; AC: Amylose content; QTL: Quantitative trait loci; CSSLS: Chromosome segment substitution lines; SSRG: Starch synthesis related gene; GBSS: Granule-bound starch synthase; ALK: Alkali degeneration; SS: Starch synthase;

GT: Gelatinization temperature; DBE: Debranching enzyme; ISA: Isoamylases; SBE: Starch branching enzyme; SSS: Soluble starch synthase; PUL: Pullulanase; AAC: Apparent amylose content; PaT: Pasting temperature; PeT: Peak time; SB: Setback viscosity; PV: Peak viscosity; FV: Final viscosity; BD: Breakdown viscosity; TV: Trough viscosity; RH: Relative humidity; ADPlar: Adenosine diphosphate glucose pyrophosphorylase large subunit 1;

ADPsma: Adenosine diphosphate glucose pyrophosphorylase small subunit; ADPiso: Adenosine diphosphate glucose pyrophosphorylase large subunit 2; GC: Gel consistency; Dul: Dull; UTR: Untranslated region

\section{Supplementary Information}

The online version contains supplementary material available at https://doi. org/10.1186/s12864-021-07913-7.

Additional file 1: Table S1. Correlation analysis of seven RVA parameters in 2013 and 2014. Table S2. Correlation analysis of seven RVA parameters in 2016 and 2017. Table S3. Correlation analysis of starch RVA parameters

Additional file 2: Table S4. Genotype and phynotype of CSSLS. Additional file 3. Sequence Alignments of SSRGs between 9311 and Nipponbare.

\section{Acknowledgements}

We thank Dr. Wunyin Zhu and Miss Jin Lin for their works during the development of CSSLS.

Authors' contributions

$L Z, C Z$ and $C W$ designed the experiments. $L Z$ and SY prepared powers of the grain and measured the RVA profile. LZ, TC, QZ, ZZ, YZ developed the CSSLs populations and CZ identified the CSSLs by molecular markers. TC and ZZ managed the planting of materials in field. QZ performed analyses. LZ wrote the manuscript which was modified by $Y Z$. All authors read and approved the final manuscript.

\section{Funding}

This work was supported by grants from the Natural Science Foundation of Jiangsu Province of China (BK20180302), the Agriculture Research System of China (CARS-01-62), Jiangsu Agriculture Science and Technology Innovation Fund (CX(18)1001), and the Provincial Key Laboratory of Agrobiology, Jiangsu Academy of Agricultural Sciences, China. The funding bodies played no role in the design of the study, collection, analysis, and interpretation of data and in writing the manuscript.

\section{Availability of data and materials}

All materials analyzed during the current study are available from the Jiangsu Provincial Platform for Conservation and Utilization of Agricultural

Germplasm (http://jagis.jaas.ac.cn/CL_crop.aspx). The stock numbers for 9311 and Nipponbare are M3A00407620 and SD_NJAU_B149, respectively. The results of correlation analysis among RVA parameters in different years are in the Additional file (1) The stock numbers, raw phenotype data and genotype data for all CSSLs are in Additional file (2) The details about the sequence alignments are in the additional file 3.

\section{Declarations}

Ethics approval and consent to participate Not applicable. 


\section{Consent for publication}

Not applicable.

\section{Competing interests}

The authors declare that they have no competing interests.

\section{Author details}

'Institute of Food Crops, Jiangsu Academy of Agricultural Sciences, Jiangsu High Quality Rice R\&D Center, Nanjing Branch of China National Center for Rice Improvement, Nanjing 210014, China. ${ }^{2}$ Jiangsu Collaborative Innovation Center for Modern Crop Production, 210095 Nanjing, China.

Received: 28 December 2020 Accepted: 26 July 2021

Published online: 05 August 2021

\section{References}

1. Tong C, Chen Y, Tang F, Xu F, Hung Y, Cheng H, et al. Genetic diversity of amylose content and RVA pasting parameters in 20 rice accessions grown in Hainan, China. Food Chem. 2014;161:239-45.

2. Cozzolino D. The use of the rapid visco analyser (RVA) in breeding and selection of cereals. J Cereal Sci. 2016;70:282-90.

3. Yang Y, Ni D, Song F, Li L, Lu X, Li Z, et al. Identification of QTLs for rice starch RVA profile properties in different ecological sites. Acta Agronomica Sinica. 2012;38(2):264-74 (in Chinese with English abstract).

4. Vk BR, Ns C. Deploying viscosity and starch polymer properties to predict cooking and eating quality models: a novel breeding tool to predict texture. Carbohyd Polym. 2021;260:117766.

5. Mao T, Zhang Z, Ni S, Zhao Y, Li X, Zhang L, et al. Assisted selection of eating quality progeny of indica (O. sativa L. ssp. indica) and japonica (O. sativa L. ssp. japonica) hybrids using rice starch properties. Genet Resour Crop Evol. 2021;68:411-20.

6. Yan C, Tian Z, Fang Y, Yang Y, Li J, Zeng S, et al. Genetic analysis of starch paste viscosity parameters in glutinous rice Oryza sativa L. Theor Appl Genet. 2011;122:63-76

7. Bao J, Zheng X, Xia Y, He P, Shu Q, Lu X, et al. QTL mapping for the paste viscosity characteristics in rice (Oryza sativa L.). Theor Appl Genet. 2000;100: 280-4.

8. Lapitan V, Redona E, Abe T, Brar D. Mapping of quantitative trait loci using a double-haploid population from the cross of indica and japonica cultivars of rice. Crop Sci. 2009;49:1620-8.

9. Zhang C, Chen S, Ren X, Lu Y, Liu D, Cai X, et al. Molecular structure and physicochemical properties of starches from rice with different amylose contents resulting from modification of OsGBSI activity. J Agr Food Chem. 2017;65(10):2222-32.

10. Su Y, Rao Y, Hu S, Yang Y, Gao Z, Zhang G, et al. Map-based cloning proves qGC-6, a major QTL for gel consistency of japonica/indica cross, responds by Waxy in rice (Oryza sativa L.). Theor Appl Genet. 2011;123:859-67.

11. Bao J, Sun M, Zhu L. Corke H. Analysis of quantitative trait locus for some starch properties in rice (Oryza sativa L.), thermal properties, gel texture, swelling volume. J Cereal Sci. 2004;39:379-85.

12. Fan C, Yu X, Xing Y, Xu C, Luo L, Zhang Q. The main effects, epistatic effects and environmental interactions of QTLs on the cooking and eating quality of rice in a doubled-haploid line population. Theor Appl Genet. 2005;110: $1445-52$.

13. Umemoto T, Yano M, Satoh H, Shomura A, Nakamura Y. Mapping of a gene responsible for the difference in amylopectin structure between japonicatype and indica-type rice varieties. Theor Appl Genet. 2002;104:1-8.

14. Tian Z, Quian Q, Liu Q, Yan M, Liu X, Yan C, et al. Allelic diversities in rice starch biosynthesis lead to a diverse array of rice eating and cooking qualities. Proc Natl Acad Sci USA. 2009;106:21760-5.

15. Xu F, Bao J, He Q, Park Y. Genome-wide association study of eating and cooking qualities in different subpopulations of rice (Oryza sativa L.). BMC Genom. 2016;17:663.

16. Xiang X, Kang C, Xu S, Yang B. Combined effects of Wx and SSIlla haplotypes on rice starch physicochemical properties. J Sci Food Agri. 2017; 97:1229-34.

17. Yang F, Chen Y, Tong C, Huang Y, Xu F, Li K, et al. Association mapping of starch physicochemical properties with starch synthesis-related gene markers in nonwaxy rice (Oryza sativa L.). Mol Breeding. 2014;34:1747-63.
18. Zhang Q, Zhan Y, Zhu Z, Zhao L, Zhao Q, Xu L, et al. Inheritance analysis and QTL mapping of rice starch viscosity (Rapid Visco Analyzer profile) characteristics. Rice Sci. 2008;15:186-94.

19. Liu X, Wan X, Ma X, Wan J. Dissecting the genetic basis for the effect of rice chalkiness, amylose content, protein content, and rapid viscosity analyzer profile characteristics on the eating quality of cooked rice using the chromosome segment substitution line population across eight environments. Genome. 2010;54:64-80.

20. Zhu W, Lin J, Yang D, Zhao L, Zhang Y, Zhu Z, et al. Development of chromosome segment substitution lines derived from backcross between two sequenced rice cultivars, indica recipient $93-11$ and japonica donor Nipponbare. Plant Mol Biol Rep. 2009;27:126-31.

21. Zhang Y, Jiang L, Liu X, Liu S, Chen L, Zhai H, et al. Analysis of QTLs for starch RVA profile properties in the superior rice cultivar Koshihikari. China J Rice Sci. 2010;24(2):137-44. (in Chinese with English abstract).

22. Zhang C, Bing H, Zhu K, Zhang H, Leng Y, Tang S, et al. QTL mapping for rice RVA properties using high-throughput resequenced chromosome segment substitution lines. Rice Sci. 2013;20(6):407-14.

23. Liu X, Zhu K, Zhang C, Hong R, Sun P, Tang S, et al. Mapping of minor QTLs for rice gelatinization temperature using chromosome segment substitution lines from indica 9311 in the japonica background. Acta Agronomica Sinica. 2014;40(10):1740-7. (in Chinese with English abstract).

24. Yao X, Wang J, Liu J, Zhang J, Ren C, Ma D, et al. Mapping quantitative trait loci associated with starch paste viscosity in rice (Oryza sativa L.) under different environmental conditions. Plant Breed. 2017;136:591-602.

25. Shar T, Sheng Z, Ali U, Fiaz S, Wei X, Xie L, et al. Mapping quantitative trait loci associated with starch paste viscosity attributes by using double haploid populations of rice (Onyza sativa L.). J Integr Agric. 2020;19(7):1691-703.

26. Bao J, Wu Y, Hu B, Wu P, Cui H, Shu Q. QTL for rice grain quality based on a $\mathrm{DH}$ population derived from parents with similar apparent amylose content. Euphytica. 2002;128:317-24.

27. Yan B, Tondi Y, Chen J, Wang Y, Gao G, Zhang Q, et al. Analysis of minor quantitative trait loci for eating and cooking quality traits in rice using a recombinant inbred line population derived from two indica cultivars with similar amylose content. Mol Breed. 2014;34:2151-63.

28. Lu F, Park Y. Sequence variations in OsAGPase significantly associated with amylose content and viscosity properties in rice (Oryza sativa L.). Genet Res. 2012;94:179-89.

29. Yu J, Buckler E. Genetic association mapping and genome organization of maize. Curr Opin Biotech. 2006;17(2):155-60.

30. Xu J, Zhao Q, Du P, Xu C, Wang B, Feng Q, et al. Developing high throughput genotyped chromosome segment substitution lines based on population whole-genome re-sequencing in rice (Oryza sativa L.). BMC Genom. 2010;11:656.

31. Zhao C, Zhou L, Yu X, Zhao Q, Chen T, Yao S, et al. Identification of tiller angle quantitative trait loci based on chromosome segment substituted lines and high-density physical map in rice. Bull Botany. 2012;47(6):1-8. (in Chinese with English abstract).

32. Yu J, Hu SN, Wang J, Wong G, Li S, Liu B, et al. A draft sequence of the rice genome (Oryza sativa L. ssp. indica). Science. 2002;296:79-92.

33. Goff SA, Ricke D, Lan TH, Presting G, Wang R, Dunn M, et al. A draft sequence of the rice genome (Oryza sativa L. ssp. japonica). Science. 2002; 296:92-100.

34. Zhang J, Zheng L, Cai Y, You X, Kong F, Wang G, et al. Correlation analysis and QTL mapping for starch RVA profile properties and amylose and protein contents in rice. China J Rice Sci. 2017;31(1):31-9. (in Chinese with English abstract).

35. Shao X, Chen Z, Liu Z, Ding C, Tang S, Li G, et al. QTL mapping for rice RVA properties using chromosome segment substitution lines derived from a cross between Koshihikari and 9311. Mol Plant Breed. 2015;2:261-8. (in Chinese with English abstract).

36. Chen L, Yan X, Wang L, Gao W, Yang J, Chen S, et al. Pasting properties, grain-filling characteristics and allelic variation linked to the grain quality in diverse rice. Euphytica. 2017;213:29-44.

37. Hsu Y, Tseng M, Wu Y, Lin M, Wei F, Hwu K, et al. Genetic factors responsible for eating and cooking qualities of rice grains in a recombinant inbred population of an inter-subspecific cross. Mol Breed. 2014;34:655-73.

38. Mei M, Jiang Y, Wan Z, Liu L, Ye D, Wang Y, et al. National Climate Centre. Climatic characteristics and major meteorological events over China in 2016. Meteorological Monthly. 2017;43(4):468-76. (in Chinese with English abstract). 
39. Feng A, Zeng H, Yin Y, Song Y, Liu Y, Wang Y, et al. Climatic characteristics and major meteorological events over China in 2017. Meteorological Monthly. 2018;44(4):548-55. (in Chinese with English abstract).

40. Jiang $\mathrm{H}$, Dian W, Wu P. Effect of high temperature on fine structure of amylopectin in rice endosperm by reducing the activity of the starch branching enzyme. Phytochemistry. 2003;63:53-9.

41. Lin CJ, Li CY, Lin SK, Yang FH, Huang JJ, Liu YH, Lur HS. Influence of high temperature during grain filling on he accumulation of storage proteins and grain quality in rice (Oryza sativa L.). J Agric Food Chem. 2010;58: 10545-52.

42. Zhang C, Zhou L, Zhu Z, Lu H, Zhou X, Qian Y, et al. Characterization of grain quality and starch fine structure of two japonica rice (Oryza Sativa) cultivars with good sensory properties at different temperatures during the filling stage. J Agric Food Chem. 2016;64:4048-57.

43. Hittalmani S, Huang N, Courtois B, Venuprasad R, Shashidhar H, Zhuang J, et al. Identification of QTL for growth and grain yield-related traits in rice across nine locations of Asia. Theor Appl Genet. 2003;107:679-90.

44. Bao J, Xia Y. Genetic control of the paste viscosity characteristics in indica rice (Oryza sativa L.). Theor Appl Genet. 1999;98:1120-4.

45. Wang J, Li H, Zhang L, Meng L. 2013. Users' Manual of QTL IciMapping. The Quantitative genetics group, Institute of crop science, Chinese Academy of Agricultural Sciences (CAAS), Beijing 100081, China, and Genetic Resources Program, International Maize and Wheat Improvement Center (CIMMYT), Mexico.

46. Eshed Y, Zamir D. An introgression line population of Lycopersican pennelli in the cultivated tomato enables the identification and fine mapping of yield associated QTL. Genetics. 1995;141(3):1147-62.

47. McCouch S, Cho Y, Yano M, Paul E, Blinstrub M, Morishima H, et al. Report on QTL nomenclature. Rice Genetics Newslett. 1997;14:11-3.

\section{Publisher's Note}

Springer Nature remains neutral with regard to jurisdictional claims in published maps and institutional affiliations.

Ready to submit your research? Choose BMC and benefit from:

- fast, convenient online submission

- thorough peer review by experienced researchers in your field

- rapid publication on acceptance

- support for research data, including large and complex data types

- gold Open Access which fosters wider collaboration and increased citations

- maximum visibility for your research: over $100 \mathrm{M}$ website views per year

At $\mathrm{BMC}$, research is always in progress.

Learn more biomedcentral.com/submissions 